\title{
O impacto na qualidade de vida de fisioterapeutas na linha de frente à pandemia da
}

\section{Covid-19}

\author{
The impact on the quality of life of physiotherapists in the Covid-19 pandemic front line \\ El impacto en la calidad de vida de los fisioterapeutas en la primera línea de la pandemia del
}

\section{Covid-19}

\section{Resumo}

Diante da pandemia do novo coronavírus (Covid-19), o profissional fisioterapeuta tem papel importante dentro do contexto que estamos vivendo. Além da excessiva e degradante jornada de trabalho imposta a eles, o que torna suas rotinas mais estressantes, torna-se indispensável a preocupação com a qualidade de vida do profissional envolvido. Frente a isso, esse estudo teve como objetivo relatar o impacto na qualidade de vida de fisioterapeutas na linha de frente à pandemia da Covid-19. Os objetivos específicos foram avaliar a qualidade do sono e correlacionar a qualidade de vida com a qualidade de sono de fisioterapeutas na linha de frente à pandemia da Covid-19. A população foi composta por fisioterapeutas do Hospital de Clínicas de Passo Fundo, Hospitalar Santa Terezinha de Erechim e Hospital de Caridade de Erechim, com uma amostra de treze fisioterapeutas, de ambos os sexos, com média de idade de 29,5 anos, que atuam na linha de frente do Covid-19 no ambulatório e UTI dos hospitais já mencionados. Como resultados alcançados, pode-se observar que ambos hospitais apresentaram uma auto-avaliação da qualidade de vida regular, com maiores escores no domínio meio ambiente $(71,15 \%)$ e pior no domínio físico $(48,63 \%)$. Em relação a qualidade do sono, houve uma presença de distúrbio do sono em $23,1 \%$ e qualidade do sono ruim em $76,9 \%$. Houve uma correlação negativa e moderada entre as relações sociais com a qualidade do sono, e as demais correlações 
mostraram-se bem fracas. Deste modo, conclui-se através dos resultados que os profissionais fisioterapeutas que atuam na linha de frente da Covid-19 apresentaram uma qualidade de vida regular e diminuição da qualidade do sono.

Palavras-chave: Coronavírus; Qualidade de vida; Fisioterapia.

\begin{abstract}
Faced with the pandemic of the new coronavirus (Covid-19), the professional physiotherapist has an important role within the context we are experiencing. In addition to the excessive and degrading work hours imposed on them, which makes their routines more stressful, concern with the quality of life of the professional involved is essential. In view of this, this study aimed to report the impact on the quality of life of physical therapists in the front line to the Covid-19 pandemic. The specific objectives were to assess sleep quality and correlate quality of life with the quality of sleep of physiotherapists in the front line of the Covid-19 pandemic. The population consisted of physiotherapists from the Hospital de Clínicas de Passo Fundo, Hospitalar Santa Terezinha - Erechim and Hospital de Caridade de Erechim, with a sample of thirteen physical therapists, of both sexes, with an average age of 29.5 years, who work on the front line of Covid-19 in the outpatient and ICU of the hospitals already mentioned. As results achieved, it can be seen that both hospitals had a regular self-assessment of quality of life, with higher scores in the environment domain $(71.15 \%)$ and worse in the physical domain (48.63\%). Regarding sleep quality, there was a presence of sleep disorder in $23.1 \%$ and poor sleep quality in $76.9 \%$. There was a negative and moderate correlation between social relationships and sleep quality, and the other correlations were very weak. Thus, it is concluded through the results that the physical therapists who work on the front line of Covid-19 presented a regular quality of life and decreased quality of sleep.
\end{abstract}

Keywords: Coronavirus; Quality of life; Physiotherapy.

\title{
Resumen
}

Ante la nueva pandemia de coronavirus (Covid-19), el fisioterapeuta tiene un papel importante en el contexto en el que vivimos. Además de la jornada laboral excesiva y degradante que se les impone, que hace más estresante sus rutinas, es fundamental preocuparse por la calidad de vida de los profesionales implicados. En vista de esto, este estudio tuvo como objetivo informar el impacto en la calidad de vida de los fisioterapeutas en la primera línea de la pandemia Covid-19. Los objetivos específicos fueron evaluar la calidad del sueño y correlacionar la calidad de vida con la calidad del sueño de los fisioterapeutas en la primera línea de la pandemia de Covid-19. La población estuvo conformada por fisioterapeutas del Hospital de Clínicas de Passo Fundo, Hospital Santa Terezinha de Erechim y Hospital de Charidade de Erechim, con una muestra de trece fisioterapeutas, de ambos sexos, con una edad media de 29,5 años, que laboran en el frente línea de Covid-19 en el ambulatorio y UCI de los hospitales mencionados. Como resultados alcanzados, se puede observar que ambos hospitales presentaron una autoevaluación regular de la calidad de vida, con puntuaciones más altas en el dominio ambiental $(71,15 \%)$ y peores en el dominio físico $(48,63 \%)$. En cuanto a la calidad del sueño, hubo presencia de alteración del sueño en el $23,1 \%$ y mala calidad del sueño en el $76,9 \%$. Hubo una correlación negativa y moderada entre las relaciones sociales y la calidad del sueño, y las otras correlaciones fueron muy débiles. Así, se concluye a partir de los resultados que los fisioterapeutas que trabajan en primera línea de Covid-19 tenían una calidad de vida regular y una calidad de sueño disminuida.

Palabras clave: Coronavirus; Calidad de vida; Fisioterapia.

\section{Introdução}

Em tempos de pandemia devido ao surgimento do novo coronavírus (Sars-Cov-2) o cenário mundial está passando por um momento difícil em busca de respostas e tentativas para acabar com essa situação que vem assombrando a todos. A palavra pandemia significa enfermidade epidêmica amplamente disseminada, característica agora direcionada ao Covid-19 que além de ser fatal é considerado um grande problema de saúde pública nos últimos cem anos, comparando-se apenas a gripe espanhola que matou cerca de 25 milhões de pessoas entre 1918 e 1920 (Medeiros, 2020).

Manifestou-se pela primeira vez na China no final do ano de 2019, o patógeno foi avaliado e descrito como beta coronavírus, nova pneumonia por coronavírus assim definida pela Organização Mundial da Saúde (OMS). Devido a sua rápida dispersão e fácil contagio, outros países logo manifestaram também sintomas e casos confirmados e em março de 2020 a OMS declarou que estaríamos a enfrentar uma nova pandemia (Lana et al., 2020; Silva et al., 2020).

Frente a essa pandemia, vários profissionais da saúde estão envolvidos na recuperação física dos pacientes diagnosticados com Covid-19, dentre eles, se destaca a atuação fisioterapêutica, com papel importante tanto no tratamento da doença como também na prevenção e reabilitação das complicações respiratórias e funcionais do dia a dia por ela provocadas (Silva et al., 2020). 
Se não fosse suficiente os problemas individuais relativos ao Covid-19, a cada dia o número de casos confirmados aumenta e a escassez de equipamentos de proteção individual (EPIs) torna-se uma preocupação, a sobrecarga de trabalho, falta de medicamentos específicos e sentimento de apoio inadequado geram estresse e insegurança para os profissionais da saúde (Lai et al., 2020). Além disso, a exaustão física e mental vem atingindo grande parte desses profissionais, devido a dor por perder pacientes, colegas, familiares, além de estar exposto diretamente ao vírus, podendo ser ele o transmissor para outras pessoas e familiares que residem no mesmo local e por isso é importante que esses profissionais disponham de assistência médica e psicológica para que consigam se manter na luta contra o Covid-19 (Medeiros, 2020).

Desse modo, devido ao surgimento do Covid-19 no cenário mundial e apesar da rápida propagação, é clara a necessidade de avaliar a qualidade de vida de profissionais da saúde, especialmente fisioterapeutas que estão na linha de frente no combate à pandemia.

\section{Metodologia}

Realizou-se um estudo de corte transversal com finalidade aplicada, de natureza observacional, com abordagem quantitativa, incluindo dezessete profissionais fisioterapeutas do Hospital de Caridade e Santa Terezinha de Erechim e Hospital de Clínicas de Passo Fundo. Para realização deste estudo foram incluídos fisioterapeutas que atuam na linha de frente do Covid-19 no ambulatório e UTI do Hospital Santa Terezinha de Erechim, Hospital de Caridade de Erechim e Hospital de Clínicas de Passo Fundo, que aceitaram em participar mediante assinatura do Termo de Consentimento Livre e Esclarecido (TCLE). Foram excluídos os fisioterapeutas que não encaminharam os questionários até a data estabelecida.

Após apreciação e aprovação do projeto de pesquisa sob o número 36196420.0.0000.5351 pelo Comitê de Ética em Pesquisa (CEP) da Universidade Regional Integrada do Alto Uruguai e das Missões (URI) - Erechim, as alunas pesquisadoras entraram em contato telefônico com os coordenadores responsáveis pelos fisioterapeutas que atuam na linha de frente do Covid-19 do Hospital Santa Terezinha de Erechim, Hospital de Caridade de Erechim e Hospital de Clínicas de Passo Fundo, explicando o estudo e solicitando a liberação da realização do mesmo. Após, autorizados pelos fisioterapeutas, os coordenadores repassaram os dados telefônicos dos fisioterapeutas, e por sua vez as pesquisadoras entraram em contato telefônico com os mesmos para o convite em participar da pesquisa, juntamente com explicações sobre os objetivos do estudo. Posteriormente, foi encaminhado um formulário do google docs via whatsapp, contendo o TCLE, para assinatura daqueles que concordaram em participar da pesquisa e em seguida foi enviado via whatsapp os questionários de qualidade de vida WhoqolBref: sendo esse composto por 26 perguntas (sendo a pergunta número 1 e 2 sobre a qualidade de vida geral), as respostas seguem a escala de Likert (de 1 a 5 , quanto maior a pontuação melhor a qualidade de vida), sendo os resultados apresentados em \% de 0 a 100, ou seja, quanto maior o percentual maior é a qualidade de vida. Além dessas duas questões (1 e 2), o instrumento tem 24 facetas as quais compõem 4 domínios que são: físico, psicológico, relações sociais e meio ambiente. E o questionário Qualidade de Sono de Pittsburgh (PSQI): que é um instrumento genérico desenvolvido com o propósito de avaliar a qualidade de sono com relação ao último mês. De outro modo, ele permite avaliar qualitativamente e quantitativamente o sono. É um questionário acessível e simples de ser respondido, capaz de discriminar os pacientes entre "bons dormidores" e "maus dormidores". As questões do PSQI estão distribuídas em sete domínios, aos quais podem ser atribuídos de zero a três pontos. A somatória total pode chegar a 21 pontos, sendo que os escores superiores a 5 pontos indicam padrão ruim de qualidade de sono.

Para tratamento dos dados foi realizada análise descritiva por meio da utilização do Excel. Os dados foram apresentados de forma descritiva simples, onde as variáveis quantitativas foram apresentadas por meio de média, desvio padrão, valores mínimos, valores máximos e frequências relativas (percentuais) através de gráficos e tabelas, também foi utilizado a correlação de Pearson para correlacionar os valores da Qualidade de Vida e Qualidade do Sono. 


\section{Resultados e Discussão}

No presente estudo, foram recrutados treze profissionais fisioterapeutas que atuam na linha de frente da Covid-19, três do Hospital de Caridade e dois do Hospital Santa Terezinha e 8 do Hospital de Clínicas de Passo Fundo, sendo 80\% do sexo feminino e $20 \%$ do sexo masculino, com média de idade de 29,15 \pm 4,09 anos. Dessa amostra, 40\% foram contaminados pela Covid-19. Os sintomas relatados pelos profissionais contaminados foram: anosmia, ageusia, cefaleia, dor de garganta, fadiga, febre, mialgia, tosse seca, perda de apetite, dispneia e diarreia.

Pesquisas atuais relatam que mulheres parecem ser mais afetadas por disfunções olfativas e gustativas do que os homens. Heidari et al. (2020) em seu estudo multicêntrico, avaliaram 417 pacientes com Covid-19 leve a moderada, realizado por meio de entrevista telefônica/questionário verbal, para indivíduos que apresentaram queixa de disfunção olfatória, sendo constatada em 85,6\% dos casos ( $\mathrm{n}=357)$. Destes, 284 (79,6\%) referiram anosmia, enquanto 73 (20,4\%) hiposmia. Além do mais, a fantosmia e a parosmia afetaram $12,6 \%$ e $32,4 \%$ dos pacientes, respectivamente.

Considera-se os sinais e sintomas da Covid-19 mais comuns, a febre (temperatura $\geq 37,8^{\circ} \mathrm{C}$ ), tosse, dispneia, mialgia e fadiga (Ministério da Saúde, 2020). Huang (2020) realizou uma pesquisa com 41 casos confirmados na China e identificaram que a febre esteve presente em $98 \%$ deles, seguida por tosse (76\%), dispneia (55\%) e mialgia/fadiga (44\%). Já no estudo de Wang et al. (2020) descreveram 69 casos da China e 15\% dos indivíduos analisados obtiveram a tríade febre, tosse e dispneia. Da mesma forma que um relato de casos de Seattle, nos Estados Unidos, mostrou que 50\% deles não manifestaram febre, sendo a tosse e a dispneia os sintomas mais relatados, por $88 \%$ das pessoas (Bhatraju, 2020).

Clemency et al. (2020) em seu estudo composto por 961 profissionais de saúde, identificaram que 225 (23\%) foram contaminados pelo vírus. A perda do paladar ou do olfato foi o sintoma com maior razão de verossimilhança positiva $(3,33)$, corroborando com os dados encontrados do presente estudo. Além disso, a tosse seca, independentemente da presença ou ausência de outros sintomas, foi o sintoma mais sensível (74\%) e o menos específico (32\%).

O objetivo geral do presente estudo foi relatar o impacto na qualidade de vida de fisioterapeutas que atuam na linha de frente à pandemia da Covid-19 em Erechim e Passo Fundo, através da aplicação do Questionário Whoqol-bref, sendo avaliado a auto-avaliação da Qualidade de Vida, a qual engloba a percepção de Qualidade de Vida e Satisfação com a Saúde e os Domínios Físico, Psicológico, Relações Sociais e Meio Ambiente. Esses resultados serão apresentados por meio de estatística descritiva simples, através de média, desvio padrão, valor mínimo e valor máximo.

Inicialmente foram analisados os resultados referentes às duas questões gerais, que tratam da percepção que o indivíduo tem de sua qualidade de vida e de sua saúde. A primeira questão avaliou a percepção subjetiva do indivíduo sobre a sua qualidade de vida geral. Identificou-se que $66,23 \%$ dos profissionais dizem ter boa qualidade de vida, 23,07\% nem ruim, nem boa e 7,7\% ruim, assim como a segunda questão que avaliou a percepção do indivíduo sobre a sua saúde. Destes $69,23 \%$ dizem estar satisfeitos, nem satisfeito, nem insatisfeitos, e insatisfeito apresentaram um percentual de 15,38\% cada.

As médias do Questionário Whoqol-bref de ambos Hospitais de Erechim e de Clínicas de Passo Fundo, revelaram uma auto-avaliação de Qualidade de Vida Regular. Em relação aos quatro domínios da QV, em uma escala de 0 a 100, todos os domínios, com exceção do domínio Físico tiveram média geral acima de 60, indicando uma boa Qualidade de Vida. Pode-se constatar que o Domínio Meio Ambiente apresentou um escore maior enquanto o Domínio Físico um escore menor, com valores de $15,38 \pm 1,95$, representando $71,15 \%$ e $11,78 \pm 1,95$, representando $48,63 \%$ respectivamente, ilustrados no Gráfico 1 e Tabela 1. 
Gráfico 1: Resultados do Questionário Whoqol-bref dos Fisioterapeutas do Hospital de Caridade e Santa Terezinha de Erechim e Hospital de Clínicas de Passo Fundo.

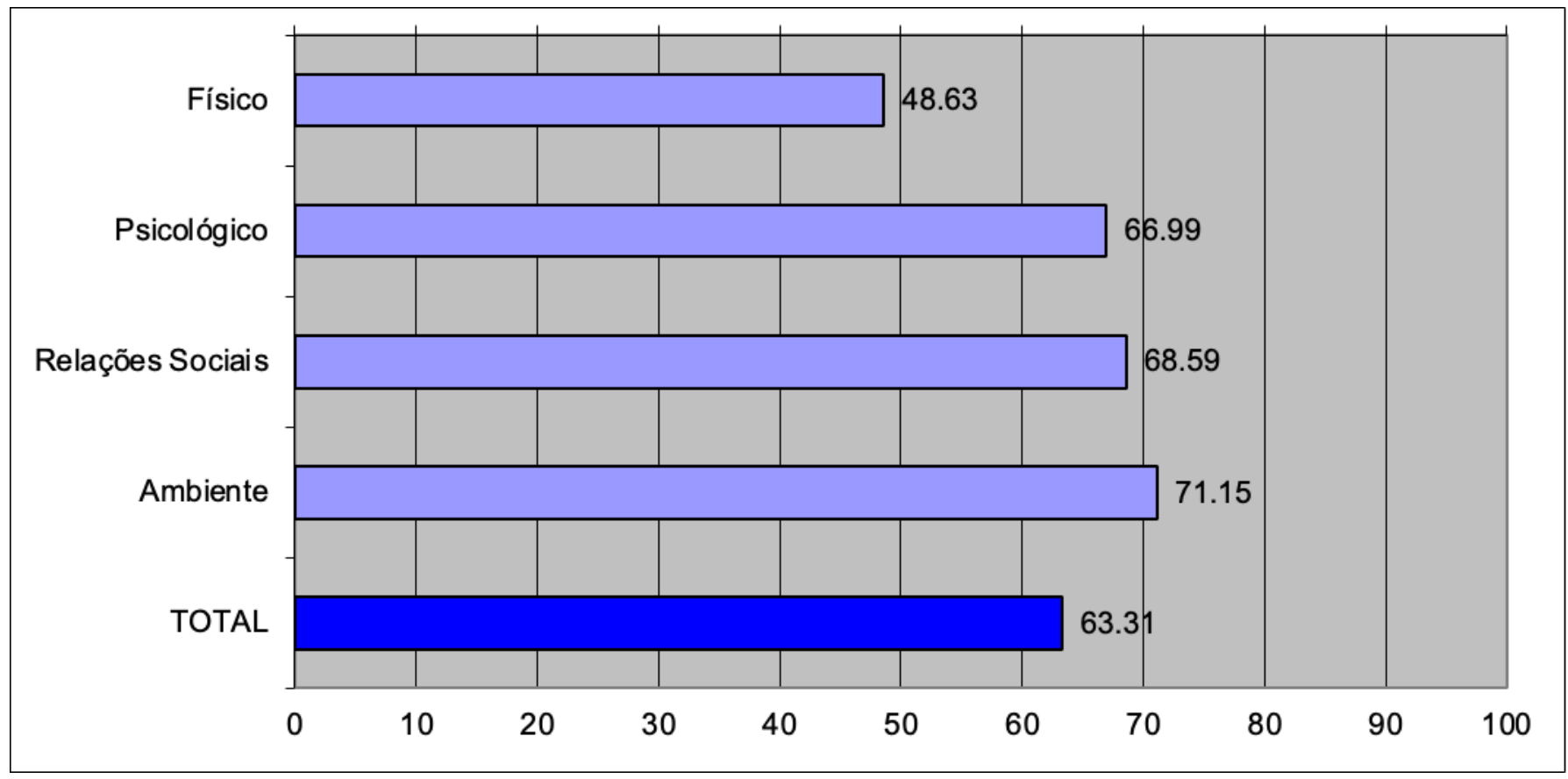

Fonte: Autores (2020).

Tabela 1: Resultados das Médias, Desvio Padrão (DP), Valor Mínimo e Valor Máximo do Questionário Whoqol Bref:

\begin{tabular}{l|c|c|c|c|c|c}
\hline \multicolumn{1}{c|}{ DOMÍNIO } & MÉDIA & $\begin{array}{c}\text { DESVIO } \\
\text { PADRÃO }\end{array}$ & $\begin{array}{c}\text { COEFICIENTE } \\
\text { DE VARIAÇÃO }\end{array}$ & $\begin{array}{c}\text { VALOR } \\
\text { MÍNIMO }\end{array}$ & $\begin{array}{c}\text { VALOR } \\
\text { MÁXIMO }\end{array}$ & AMPLITUDE \\
\hline Físico & 11,78 & 1,95 & 16,51 & 8,57 & 14,86 & 6,29 \\
Psicológico & 14,72 & 1,67 & 11,32 & 10,67 & 17,33 & 6,67 \\
Relações Sociais & 14,97 & 3,58 & 23,88 & 6,67 & 20,00 & 13,33 \\
Meio Ambiente & 15,38 & 1,95 & 12,67 & 11,50 & 18,00 & 6,50 \\
Auto-avaliação da QV & 14,31 & 2,81 & 19,64 & 8,00 & 16,00 & 8,00 \\
\hline TOTAL & $\mathbf{1 4 , 1 3}$ & $\mathbf{1 , 7 4}$ & $\mathbf{1 2 , 3 1}$ & $\mathbf{1 0 , 7 7}$ & $\mathbf{1 6 , 3 1}$ & $\mathbf{5 , 5 4}$ \\
\hline
\end{tabular}

Fonte: Autores (2020).

Souza e Stancato (2020) realizaram um estudo transversal que contou com participação de 116 profissionais da saúde, que trabalhavam nos plantões matutino e vespertino, com idade média de 35 anos e com predomínio do sexo feminino $(78,8 \%)$. A profissão predominante foi de técnicos de enfermagem $(51,4 \%)$. Considerou-se boa a qualidade de vida em $80 \%$ quando em relação à saúde, tendo 62,9\% de ausência de problemas atuais de saúde, diante disso, as médias dos domínios do Whoqol-bref foram: domínio físico, 68,1\%; domínio psicológico, 63,7\%. Os domínios ambiental e relações sociais obtiveram $55,2 \%$ e $59,6 \%$ respectivamente.

Foi então possível observar que Wang e Zhang juntamente com seus colaboradores (2020) identificaram através de seus estudos que a população em geral, diante da pandemia apresentou sintomas mais frequentes de depressão, ansiedade e estresse, sendo ainda mais relevante estes sintomas em profissionais da saúde. No presente estudo, 50\% apresentaram sentimentos negativos, como mau humor, desespero, ansiedade e depressão com muita frequência, no entanto o domínio psicológico indicou boa qualidade de vida. 
Pesquisas alertam para o sentimento de impotência que alguns profissionais da saúde enfrentam diante da gravidade e a complexidade dos casos frente a falta de leitos ou equipamentos de suporte a vida. Por isso Teixeira et al. (2020) realizaram um estudo onde foi possível constatar que além do transtorno de ansiedade generalizada, verificou-se a presença de estresse crônico, exaustão e/ou o esgotamento dos trabalhadores que enfrentam uma acentuada carga de trabalho, devido a possibilidade do surgimento de escassez de mão-de-obra, na possibilidade dos profissionais de saúde necessitarem de isolamento, devido ao fato de contraírem o vírus Sars-Cov-2.

No estudo realizado por Zhang et al. (2020) com 1.563 médicos participantes, que atuavam em hospitais de diferentes cidades chinesas, detectou-se a prevalência de sintomas de estresse em 73,4\% dos respondentes, depressão em 50,7\%, ansiedade em 44,7\%, e insônia em 36,1\%. Em referência ao estresse e à insônia, em particular, é provável a ocorrência de um círculo vicioso, em que as dificuldades para dormir aumentavam os níveis de estresse e vice-versa.

O temor de ser infectado, a proximidade com o padecimento dos pacientes ou a morte destes, assim como a angustia dos familiares relacionada com a falta de suprimentos médicos, incertezas sobre diversos recursos, isolamento e preocupações com entes queridos foram aspectos abordados no estudo de Huang et al. (2020) e também relatados em outro trabalho que discorreu sobre o sofrimento psíquico e o adoecimento mental dos profissionais de saúde, acarretando até mesmo em alguns casos a relutância em trabalhar.

O primeiro objetivo específico foi avaliar a qualidade de sono de fisioterapeutas que atuam na linha de frente à pandemia da Covid-19 em Erechim e Passo Fundo. Para tal, foi utilizado o Índice de Qualidade de Sono de Pittsburgh, que possibilitou a verificação da qualidade do sono dos profissionais da linha de frente à pandemia da Covid-19 no último mês. Este instrumento é composto por sete componentes distintos, porém que se correlacionam. Para melhor visualização dos resultados seus componentes foram analisados separadamente e ao final uma representação do escore global. A análise estatística utilizada para esta variável foi simples através de média e percentual.

A percepção dos profissionais sobre a qualidade do sono no último mês, ficando este com um percentual considerável entre $61,5 \%(n=8)$ ruim e $38,5 \%(n=5)$ muito ruim.

No componente sobre latência do sono, no item tempo para início do sono, 61,5\% declararam passar 15 minutos ou menos para iniciar o sono, 15,4\% entre 16 a 30 minutos, $15,4 \%$ entre 31 a 60 minutos e 7,7\% acima de 60 minutos.

Com relação à dificuldade para adormecer 7,7\% declararam não ter problemas para adormecer, 38,5\% menos de uma vez por semana, 30,8\% demoraram para adormecer em até 30 minutos no último mês uma ou duas vezes por semana e $23 \%$, três ou mais vezes na semana.

Na Duração do Sono, 23\% revelaram dormir mais que 7 horas, 30,8\% entre 6 a 7 horas e 46,1\% responderam que dormem entre 5 e 6 horas.

A eficiência habitual do sono que refere-se à relação entre o número de horas dormidas e o número de horas em permanência no leito, não necessariamente dormindo. Os resultados apontam um percentual de 46,1\%, uma eficiência superior a $85 \%, 38,5 \%$ entre 75 a $84 \%$ e $15,4 \%$, uma eficiência de 65 a $74 \%$.

Alguns dados obtidos no componente sobre distúrbios do sono mostram um pouco das perturbações, sendo que 38,4\% não acordaram nenhuma vez no meio da noite ou de manhã cedo no último mês, 23,1\% menos de uma vez por semana, 15,4\% uma ou duas vezes por semana e $23,1 \%$ três ou mais vezes na semana.

Na nossa amostra, 53,8\% não levantaram para ir ao banheiro nenhuma vez e 46,2\% levantaram menos de duas vezes. Com relação a problemas respiratórios durante o sono, a maioria, ou seja, 92,3\% relataram não ter apresentado dificuldade para respirar nenhuma vez por semana e 7,7\%, menos de uma vez por semana. $84,6 \%$ relataram não ter tosses ou roncos fortes nenhuma vez por semana e $15,4 \%$, menos de uma vez por semana. 
Com relação aos fatores ambientais que interferiram na qualidade do sono no último mês, 76,9\% não sentiram frio, e 23,1\% menos de uma vez por semana. Já para o calor, em igual percentual, ou seja, 46,1\% e 75\% não sentiram nenhuma vez por semana e menos de uma vez por semana e 7,7\% entre uma e duas vezes por semana, estes dados revelam que dentre estas questões, a sensação de calor foi a que mais dificultou a qualidade do sono.

Na nossa amostra, $84,6 \%$ não tiveram queixa de dor, $7,7 \%$ menos de uma vez por semana e $7,7 \%$ entre uma e duas vezes por semana. $38,5 \%$ não tiveram pesadelos, $53,8 \%$ tiveram pesadelos menos de uma vez por semana e 7,7\% entre uma ou duas vezes por semana.

Cerca de 61,5\% dos profissionais relataram não ter apresentado nenhuma outra razão que dificultasse o sono, além das apresentadas anteriormente. Porém dos 23,1\% afirmaram apresentar outras razões menos de uma vez por semana e 15,4\% entre uma ou duas vezes por semana. De uma maneira geral, 61,5\% dos profissionais classificaram sua qualidade do sono boa e $38,5 \%$ ruim.

Em relação a necessidade do uso de medicamentos para dormir, 61,5\% dos profissionais não necessitam de medicamentos para dormir e, 38,5\% menos de uma vez por semana, necessitando assim fazer uso de alguma medicação para favorecer o sono.

No componente sobre disfunção diurna, no que se refere à dificuldade em ficar acordado durante as atividades habituais, 46,1\% relataram não ter apresentado esse padrão nenhuma vez no último mês, 38,4\% apresentaram ao menos uma vez por semana e 15,4\% uma ou duas vezes por semana dificuldade em se manter acordado, com sua atenção plena na realização de suas atividades cotidianas.

Relacionando à dificuldade em manter o ânimo ou entusiasmo na realização das atividades habituais no último mês, obtivemos um percentual de $15,4 \%$ no que se refere a nenhuma dificuldade, $30,7 \%$, problema leve, $46,1 \%$ moderada e $7,7 \%$ muita indisposição e falta de ânimo para realização das atividades cotidianas.

$30,7 \%$ dos profissionais declararam não ter parceiro, esposo ou colega de quarto, 15,4\% parceiro ou colega, mas em outro quarto e 53,8\% responderam que tem parceiro na mesma cama.

Dentre os que possuem parceiro de quarto, $76,9 \%$ relataram que este parceiro não ouviu ronco forte nenhuma vez no último mês, $7,7 \%$, roncos menos de uma vez por semana e $15,4 \%$ uma ou duas vezes por semana. $100 \%$ comentaram não haver longas paradas na respiração enquanto dormia nenhuma vez no último mês. Com relação a contrações ou puxões nas pernas $76,9 \%$ dos profissionais não apresentaram este padrão, $15,3 \%$ menos de uma vez por semana e 7,7\% três ou mais vezes por semana.

No que se refere à apresentação de episódios de desorientação ou de confusão durante o sono, 84,6 \% não apresentaram este padrão nenhuma vez, 15,4 \% menos de uma vez por semana.

O escore Global do Índice de Qualidade de Sono de Pittsburgh, revelou uma presença de distúrbio do sono em 23,1\% e qualidade do sono ruim em maior percentual, ou seja, 76,9\%. A média final ficou em 9,6 revelando uma qualidade de sono ruim.

Silva et al. (2018) obtiveram resultados semelhantes ao presente estudo, em sua pesquisa sobre o índice de qualidade do sono de Pittsburg (PSQI), que contou com 16 enfermeiros, 50\% do turno diurno e $50 \%$ do turno noturno. Os resultados foram qualidade de sono ruim para ambos os turnos, porém, com mais destaque no turno noturno.

Ainda sobre o trabalho de Viana et al. (2020) a qualidade do sono analisada pelo Índice de Qualidade do sono de Pittsburg (PSQI), numa escala de 0 (melhor qualidade) a 21 (pior qualidade), obteve uma média de 6,8 (DP 3,39), sendo que os do turno noturno tiveram sono de qualidade pior do que aqueles que trabalhavam no turno diurno $(p=0,017)$.

Um trabalho sobre o índice da qualidade do sono foi aplicado a 257 participantes, sendo 129 da linha de frente e 128 que não faziam parte da linha de frente. Evidenciou-se que profissionais da linha de frente pontuaram mais em problemas 
relacionados a qualidade do sono, porém os resultados não foram significativos. Por exemplo, profissionais da linha de frente, $75 \%$ dormiam mal e $85 \%$ tinham estresse moderado e grave. Os profissionais que não eram da linha de frente $76 \%$ dormiam mal, e $84 \%$ estresse moderado e grave. O estudo também pontuou que o sexo feminino e experiência profissional foram os maiores índices de má qualidade do sono e estresse (Jahrami, et al, 2020).

Mediante ao estudo de transversal de Viana e seus colaboradores (2020) foram recrutados 104 enfermeiros de um Hospital Universitário. Os dados foram coletados por meio do Questionário de Informações Pessoais e Profissionais, Índice de qualidade de sono de Pittsburgh e Questionário de Whoqol-Bref. No que se refere à avaliação gráfica, em ambos os turnos, os profissionais que obtiveram boa qualidade do sono indicam um melhor resultado para qualidade de vida no domínio físico. Evidencia-se que não existe semelhança entre o padrão do sono e o domínio de qualidade da vida, apresentando-se com um resultado melhor para a qualidade de vida dos profissionais que têm melhor qualidade do sono.

Um estudo transversal de 1.257 profissionais de saúde em 34 hospitais equipados com clínicas ou enfermarias para pacientes com Covid-19 em diversas regiões da China, constatou-se que uma proporção considerável de profissionais de saúde relataram sintomas como, depressão, ansiedade, insônia, e angústia, principalmente do sexo feminino, enfermeiras, que moravam em Wuhan e profissionais de saúde que estão diretamente comprometidos no diagnóstico, tratamento ou prestação de cuidados de enfermagem a pacientes com suspeita ou confirmação de Covid-19 (Lai et al.,2020).

A prevalência da má qualidade do sono fica evidente na maior parte dos relatos recentes com profissionais da saúde, como revelados no presente estudo. Machi et al. (2012) relataram que 31\% dos 334 médicos americanos que trabalhavam em salas de emergência e participaram da pesquisa, relataram problemas com o sono. O que também foi verificado no estudo de Surani et al. (2015) onde 37\% dos 350 médicos indicaram problemas com o sono. Xiao et al. (2020), por sua vez, em uma pesquisa com 180 equipes médicas na China demonstrou elevação do PSQI de 8,6 \pm 4,6, o que se compara ao nosso estudo onde o PSQI ficou de 9,6.

Com objetivo de correlacionar a qualidade de vida e o índice qualidade do sono de Pittsburgh de fisioterapeutas na linha de frente à pandemia da Covid-19, utilizou-se a análise descritiva de Correlação de Pearson (0,00-0,19: bem fraca; 0,200,39: fraca; 0,4-0,69: moderada; 0,7-89: forte e 0,9-1,00: muito forte), onde foi possível avaliar as seguintes correlações: auto avaliação de qualidade de vida e qualidade de sono ( $\mathrm{r}=0,008708)$, domínio físico e qualidade do sono ( $\mathrm{r}=-0,03569)$, domínio psicológico e qualidade do sono ( $r=-0,15669)$, relações sociais e qualidade do sono ( $r=-0,48555)$, e meio ambiente e qualidade do sono ( $\mathrm{r}=-0,15572)$. Os resultados revelaram que houve correlação negativa e moderada entre as relações sociais com a qualidade do sono, e as demais correlações mostraram-se bem fracas.

Diante de um estudo transversal de Viana et al. (2020) com 104 enfermeiros de um Hospital Universitário, mediante os seguintes instrumentos: Questionário de Informações Pessoais e Profissionais, Índice de qualidade de sono de Pittsburgh e Questionário de Whoqol-Bref. A avaliação mostrou que ambos os turnos apresentaram boa qualidade do sono indicando melhor resultado para qualidade de vida no domínio físico. Desse modo, demonstra-se que há relação entre o padrão do sono e de qualidade de vida, ou seja, os profissionais têm melhor qualidade de vida quando tem melhor qualidade do sono.

Ravagnani e Crivelaro (2010) em seu estudo sobre a qualidade do sono e percepção da qualidade vida dos profissionais de enfermagem de uma Unidade de Terapia Intensiva, relataram que 87,5 \% dos profissionais somando turno noturno e diurno afirmaram que a qualidade do sono interfere na qualidade de vida. Diante do exposto, Siqueira et al (2009) tiveram um percentual de 68,9 \% das respostas também confirmando a relação citada acima. Os entrevistados do estudo ainda relataram que trabalham muito, estando sempre indispostos e cansados, não conseguindo dormir direito.

O sono não restaurador, ou seja, não manter uma boa noite de sono, ocasiona dificuldades na realização das atividades rotineiras, na concentração, memória, algumas vezes alteração de humor e irritação, interferindo nas relações sociais (Edinger et al., 2004). 
Evidenciando a citação de Edinger et al. (2004), Pedrosa et al. (2010) em sua pesquisa também encontraram relação entre a qualidade do sono e a qualidade de vida, descrevendo que pior qualidade do sono vai diretamente influenciar na baixa qualidade de vida, corroborando com os achados do presente estudo.

\section{Considerações Finais}

Através do presente estudo concluiu-se predominância do sexo feminino (80\%) em profissionais fisioterapeutas que atuam na linha de frente da Covid-19. Da amostra total, apenas $40 \%$ foram infectados pelo vírus. Os sintomas relatados incluíram anosmia, ageusia, cefaleia, dor de garganta, fadiga, febre, mialgia, tosse seca, perda de apetite, dispneia e diarreia.

A questão sobre qualidade de vida trouxe resultados satisfatórios para avaliação da qualidade de vida, domínio físico, psicológico, relações sociais e meio ambiente indicando uma boa qualidade de vida. Observou-se uma redução nos resultados da qualidade do sono.

Conclui-se também que houve correlação negativa e moderada entre as relações sociais com a qualidade do sono, e as demais correlações mostraram-se bem fracas. Diante disso e por se tratar de um tema atual que possui poucos estudos, sugerese a realização de novas pesquisas com amostras maiores e em hospitais de diferentes realidades.

\section{Referências}

Bhatraju, P. K. et al. (2008). Covid-19 in critically Ill patients in the Seattle Region — case series. Journal New England Medicine, 382, $2012-2022$.

Clemency, B. M. et al. (2020). Symptom criteria for Covid-19 testing of heath care workers. Academic Emergency Medicine, $27,469-474$.

Edinger, J. D. et al. (2004). Derivation of research diagnostic criteria for insomnia: report of an American Academy of Sleep Medicine work group. Sleep Medicine, 27(8), 1567-1596.

Heidari, F. et al. (2020). Anosmia as a prominent symptom of Covid-19 infection. Journal Rhinology, 58(3), $302-303$.

Huang, C. et al. (2020). Clinical features of patients infected with 2019 novel coronavirus in Wuhan, China. The Lancet, 395, $497-506$.

Jahrami, H. et al. (2020). The examination of sleep quality for frontline healthcare workers during the outbreak of Covid-19. Sleep and Breathing, 01-09.

Lai, J. et al. (2020). Factors Associated With Mental Health Outcomes Among Health Care Workers Exposed to Coronavirus Disease 2019. JAMA Network Open, 3(3), 01-12.

Lana, R. M. et al. (2020). Emergência do novo coronavírus (Sars-Cov-2) e o papel de uma vigilância nacional em saúde oportuna e efetiva. Caderno de Saúde Pública - CSP, 36(3), 01-05.

Machi, M. S. et al. (2012). The relationship between shift work, sleep, and cognition in career emergency physicians. Academic Emergency Medicine, 19, 8591.

Medeiros, E. A. S. (2020). A luta dos profissionais de saúde no enfrentamento da Covid-19. Revista Acta Paulista de Enfermagem, 33, 01-05.

Ministério da Saúde. (2020). Diretrizes para Diagnóstico e Tratamento da Covid-19 - Versão 3. Publicada em 17 de abril de 2020. https://portalarquivos.saude.gov.br/images/pdf/2020/April/18/Diretrizes-Covid19.pdf.

Pedrosa, R. P. et al. (2010). Sleep quality and quality of life in patients with hypertrophic cardiomyopathy. Cardiology, 117 (3), $200-2006$.

Revagnani, J. S; Crivelaro, P. M. S. (2010). Qualidade de sono e percepção da qualidade de vida dos profissionais de enfermagem de uma unidade de terapia intensiva. 2010. Trabalho de Conclusão de Curso (Curso de Enfermagem). Centro Universitário Católico Salesiano Auxilium, São Paulo.

Silva, C. M. S. et al. (2020). Evidence-based Physiotherapy and Functionality in Adult and Pediatric patients with Covid-19. Journal of Human Growth and Development - JHGD, 30, 148-155.

Silva, E. S. et al. (2028). Avaliação da qualidade do sono e sua relação com a qualidade de vida de trabalhadores noturnos da enfermagem do hospital de são Lourenço-MG. Revista Saúde em Foco, 10, 581-590.

Souza, M. A.; Stancato, K. (2010). Avaliação da qualidade de vida de profissionais de saúde em Campinas. Revista de Atenção à Saúde, 12(49), 155-161.

Surani, A. A. et al. (2015). To Assess Sleep Quality among Pakistani Junior Physicians (House Officers): A Cross-sectional Study. Annals of Medical and Health Science Research, 5(5) 329-333.

Teixeira, C. F. S., et al. (2020). A saúde dos profissionais de saúde no enfrentamento da pandemia de covid-19. Revista Ciência e Saúde Coletiva, 25 (9), 3465-3474. 
Research, Society and Development, v. 11, n. 1, e5611124598, 2022

(CC BY 4.0) | ISSN 2525-3409 | DOI: http://dx.doi.org/10.33448/rsd-v11i1.24598

Viana, M. O. C. et al. (2020). Qualidade de vida e sono de enfermeiros nos turnos hospitalares. Revista Cubana de Enfermaria, 34(2).

Wang, Z. et al. (2020). Clinical features of 69 cases with coronavirus disease 2019 in Wuhan, China. Clinical Infectious Diseases, $71(15), 769-777$.

Xiao, H. et al. (2020). The Effects of Social Support on Sleep Quality of Medical Staff Treating Patients with Coronavirus Disease 2019 (Covid-19) in January and February 2020 in China. Medical Science Monitor, 26.

Zhang, C. et al. (2020). Survey of insomnia and related social psychological factors among medical staffs involved with the 2019 novel coronavirus disease outbreak. Journal Frontiers in Psychiatry, 11. 